\title{
Modelling and Mathematical Analysis of the Glass Eel Migration in the Adour River Estuary
}

\author{
M. Odunlami, G. Vallet* \\ UMR CNRS 5142 - IPRA BP 115564013 Pau Cedex - France
}

\begin{abstract}
In this paper we are interested in a mathematical model of migration of grass eels in an estuary. We first revisit a previous model proposed by O. Arino and based on a degenerate convection-diffusion equation of parabolic-hyperbolic type with time-varying subdomains. Then, we propose an adapted mathematical framework for this model, we prove a result of existence of a weak solution and we propose some numerical simulations.
\end{abstract}

Keywords and phrases: mathematical modelling, glass eels, degenerate convection-diffusion

Mathematics Subject Classification: 92A18, 35K65

\section{Introduction}

The European eel is a Catadromous-Diadromous fish, that is to say that it spends most of its life in fresh water and migrates to the sea to breed. Spawning occurs in the Sargasso Sea. Eels feed and are born primarily by the Gulf Stream which irrigates the eel colonization area ranging from Mauritania to the Barents Sea. Near the continental shelf, where they come at the end of the summer, eels metamorphosed into glass eels. At this stage the muscle fibres of glass eels are poorly developed limiting their ability to swim in the estuary, where they are caught.

When they enter in the estuary, the glass eels are not able to fight against the current speed (cf. Bolliet [9] and De Casamajor et al. [14]). Thus, their behavior is mainly passive and is constrained by the flow of water $u$. Moreover, since they are used to salt water, throughout their motion in the saltwater - freshwater stratification, they need to migrate at the bottom. Indeed, in estuaries, there is a sharp boundary created between the water masses, with fresh water floating on top and a wedge of saltwater on the bottom. Then, arriving in brackish water, glass eels are able to fight against a maximum current speed given in the sequel by $\hat{u}$, else they move in the vertical water column to burrow in the bottom. This is the place where we consider our study, from the mouth of the Adour river to the "Bec du gave" in the map: Figure 1.

A last rule needed to model the behavior of glass eels indicates that they are afraid of light (cf. Bardonnet et al. [5]). Then, depending on the daylight, the full moon, the new moon and the turbidity, they dive towards the bottom, or they go towards the surface.

\footnotetext{
${ }^{*}$ Corresponding author. E-mail: guy.vallet@univ-pau.fr
} 


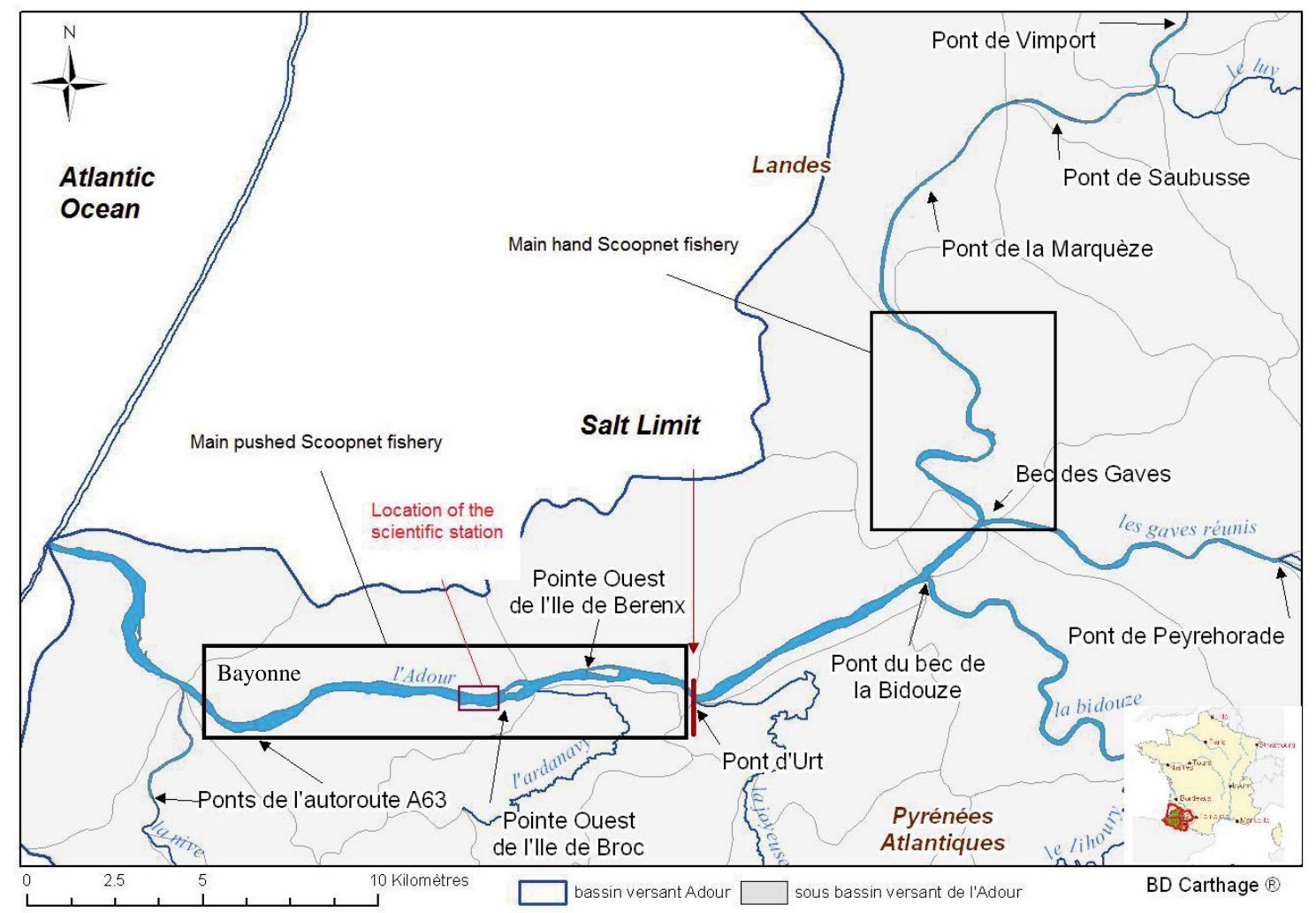

Figure 1. Map of the Adour estuary and locations of the two main glass eels fisheries (source [28]).

Fishing glass eels in the region of Bayonne (cf. map in Figure 1) is a part of the heritage of this region. Some GaveÕs fisheries are present since the eleventh century in the cartularies of some monasteries. Glass eels are also an attractive product to fishermen. Today, about $70 \%$ of annual turnover of fishermen of the Adour comes from the glass eel fishing. Now the future of this fish is uncertain. The number of glass eels has fallen sharply. This decrease is due to several factors: oceanic factors, low swimming ability of larvae, estuarine environments, É The elaboration of a robust model, aside from its scientific interest, is of direct use to the conservation of eel stocks and to improve the way that eel fisheries and habitats are managed.

In this paper we are interested in the evolution of the density of glass eels in a part of an estuary (in our case, the Adour river in the South-West of France). The initial model, proposed by O. Arino (cf. [26]) and extended by P. Prouzet et al. in the "supporting information" of paper [28], has been recently revisited by P. Prouzet et al. [27] and M. Odunlami [25] in the EELIAD project (European Eels in the Atlantic : Assessment of their Decline). To our knowledge, [26] is the only mathematical study of this type of model. In this reference, the authors propose to prove the existence of a weak solution assuming that the different sub-domains are stationary domains and they give qualitative information assuming the existence of a regular solution. Here, by the way of an extended fixed space domain and a global degenerate convection-diffusion equation with discontinuous coefficients and suitable conditions at the boundary, we obtain the good description of migration of the glass eels when coming back to the domain of evolution. By a vanishing viscosity method, we prove the existence of a weak solution and the same qualitative properties for this weak solution at the limit. 
The model is based on the semi-linear degenerate parabolic-hyperbolic problem (2.1) where $D$ is a diagonal matrix and $\boldsymbol{V}$ a vector given in (2.2). Indeed, the problem is of parabolic type when $D$ is positive-definite, it is of hyperbolic type when $D=0$; and this may occur in given time-dependant nonnegligible open sets. This corresponds to a coupling of a parabolic problem with a first order hyperbolic one, via the surfaces separating the two sets. In the case of two linear hyperbolic problems and fixed sets, there exist many papers in the literature concerning for example domain decomposition for transport equations (cf. F. Gastaldi et al. [19]). On the other hand, the nonlinear case is more delicate, see in the one dimensional case Adimurthi et al. [1], B. Andreianov et al. [4], or J. Jimenez et al. [21] for a bounded d-dimensional one.

Concerning the coupling of a second-order operator with a first order one, still for fixed domain, let us cite the paper of F. Gastaldi et al. [18] in the linear case for heat transfer studies and the paper of G. Aguilar et al. [2] for infiltration processes in an heterogeneous porous media. To our knowledge, the case of time-varying subsets has not been studied.

Realistic boundary conditions and discontinuous time-space dependence of the coefficients are the main difficulties in our study. We proposed in this paper a result of existence of a solution, but the question of the uniqueness of the solution is open. Although the main operator of the problem is linear, may be an additional condition of "entropy" type to the definition of a weak solution is needed. This is the usual condition when one proves the existence of a solution via a method of vanishing viscosity. Then, one has to consider a fully nonlinear formulation (cf. S. N. Kruvzkov [22]) and the lack of compactness and of $L^{\infty}$ a priori estimate yield to think of generalized formulations like kinetic (cf. G.-Q. Chen and B. Perthame [12]) or Young measures (cf. G. Vallet and P. Wittbold [30]) formulations, and of a generalized boundary formulation like J. Carrillo [10]. Let us also mention an other type of degenerate parabolic problems that models convection-diffusion motions in nature: M. Bendahmane et al. [7], R. Bürger [8] for Neumann conditions or G. Q. Chen et al. [11]. They are nonlinear models and, unlike our problem, they degenerate in a free set given by some values of the unknown variable.

After this brief presentation of the behavior of the animal, we expose in a second section the mathematical model. In the third section we propose the mathematical framework for this model, the definition of a solution and the result of existence of a solution. Then, in Section 4 we prove this result. The last section provides some numerical simulations of this model.

\section{The population dynamic model}

Let us, in this section, present the mathematical model for the dynamic of glass eels when they are moving in the transversal profile of the river Adour, from downstream to upstream.

The transversal profile of the river is denoted, at each time $t$, by $\widetilde{\Omega}(t)$.

If one denotes by $] L, R$ [ the base of this profile, the domain $\widetilde{\Omega}(t)$ is described by

$$
\widetilde{\Omega}(t)=\{(x, z), x \in] L, R\left[, z_{b}(x)<z<\zeta(t, x)\right\},
$$

where $z_{b}(x)$ denotes the vertical position of the bottom at abscissa $x$ in $] L, R\left[\right.$ and $\zeta(t, x)-z_{b}(x)$ the water depth as a time evolution function, because of the tide for example.

Let us denote by $C$ the glass eel density.

The model is based on the following reaction-convection-diffusion equation:

$$
\partial_{t} C-\operatorname{div}_{(\mathrm{x}, \mathrm{z})}[D \nabla C]+\operatorname{div}_{(\mathrm{x}, \mathrm{z})}(\boldsymbol{V} C)=-\mu(., C) C,
$$

where :

1. the vector $\boldsymbol{V}$ and the matrix $D$ are respectively given by

$$
\boldsymbol{V}(t, x, z)=\left(\begin{array}{c}
a(t, x, z) \\
-b(t, x, z)
\end{array}\right) \quad D(t, x, z)=\left(\begin{array}{cc}
e(t, x, z) & 0 \\
0 & d(t, x, z)
\end{array}\right)
$$


2. Glass eel mortality is given by term $\mu(t, x, z, C)=\mu_{n}(t, x, z)+\mu_{p}(t, x, z, C)$, where $\mu_{n} \geq 0$ is the coefficient of natural mortality or those becoming sedentary and $\mu_{p} \geq 0$ the coefficient of mortality due to fishery.

In order to make precise the different functions involved in the above equation, let us introduce some notations :

1. $u$ denotes the current speed. It is assumed to be a function of $(t, x)$, positive from downstream to upstream.

2. $\hat{u}$ denotes the maximum current speed allowing glass eel migration $(\hat{u}<0)$.

3. $z_{b}$, the bottom of the river, is a regular function of $x$, bounded from below by $z_{\min }$. $\zeta$, the top of the river, is a regular function of $(t, x)$, bounded from above by $z_{\max }$.

4. $h_{\min }$ denotes the depth of presence of the eels in the water column: i.e. eels mainly live in ]$z_{b}(x), \zeta(t, x)-h_{\min }(t, x)$ [. It depends on the light in the water, as a function of $(t, x)$. It is a regular function, lower bounded by a positive constant $\bar{h}$ (that means that glass eels never reach the surface).

$$
\text { Thus, } z_{\min } \leq z_{b}(x) \leq \zeta(t, x)-h_{\min }(t, x) \leq \zeta(t, x)-\bar{h}<\zeta(t, x) \leq z_{\max } .
$$

This behavior in the presence of light is explained by Bardonnet et al. in [5] and can be summarized, from experimental measurements in the Adour river, as indicated:

(a) During the day, eels stay at the bottom and $h_{\min }(t, x)=\zeta(t, x)-z_{b}(x)$.

(b) Else, it depends on the turbidity $\mathcal{T}$ in $\mathrm{NTU}^{1}$ :

i. if $\mathcal{T}>40 \mathrm{NTU}$,
A. at dusk beginning, $h_{\min }(t, x)=3 \mathrm{~m}$,
B. at dusk, $h_{\min }(t, x)=2.4 \mathrm{~m}$,
C. at night, $h_{\min }(t, x)=1.20 \mathrm{~m}$.

ii. if $8<\mathcal{T}<40 \mathrm{NTU}$,
A. at black moon with a cloudy sky, $h_{\min }(t, x)=1.5 \mathrm{~m}$;
B. at black moon with a clear sky, $h_{\min }(t, x)=1.76 \mathrm{~m}$;
C. at crescent moon with a cloudy sky, $h_{\min }(t, x)=2.02 \mathrm{~m}$;
D. at crescent moon with a clear sky, $h_{\min }(t, x)=2.28 \mathrm{~m}$;
E. at full moon with a cloudy sky, $h_{\min }(t, x)=2.54$;
F. at full moon with a clear sky, 2.80 ;

iii. if $\mathcal{T}<8 \mathrm{NTU}$,

A. at night, $h_{\min }(t, x)=2.70 \mathrm{~m}$,

B. else $h_{\min }(t, x)=\zeta(t, x)-z_{b}(x)$.

5. $H$ denotes the function of Heaviside with $H(0)=1$ to fix ideas.

6. $V_{C}$ denotes the own speed of glass eels. It will be a constant and we assume in the sequel that $\partial_{t} \zeta>-V_{C}$ (i.e. the own speed of glass eels is greater that the one of the tide).

7. $k_{h}>0$ denotes the horizontal diffusion coefficient (mainly related to current).

8. $k_{v}>0$ denotes the vertical diffusion coefficient (mainly related to glass eel behavior).

9. $f_{z}(t, x, z)=\min \left[1, \frac{\left[z-z_{b}(x)\right]^{+}}{\zeta(t, x)-z_{b}(x)}\right]$ denotes a coefficient inferring that glass eels close to the bottom of the river will move towards the bottom less quickly than the one close to the surface.

Note that, by extension, $f_{z}(t, x, z)=1$ if $z \geq \zeta(t, x)$.

10. $f_{0}(t, x, z)=1-H[u(t, x)-\hat{u}] H\left[\zeta(t, x)-h_{\min }(t, x)-z\right]$.

This function decides the vertical transport of eels towards the bottom:

(a) $f_{0}=1$ if the light is too important or if the current speed does not allow glass eel migration; and by extension if $z \geq \zeta(t, x)$. It makes them dive towards the bottom.

\footnotetext{
${ }^{1} \mathrm{NTU}$ : nephelometric turbidity unit
} 
(b) $f_{0}=0$ otherwise and glass eels may move towards the surface.

11. $\mu_{p}(t, x, z, C)=\hat{\mu}(C)\left(1-f_{0}(t, x, z)\right) H[z-(\zeta(t, x)-\omega)]$ where $\hat{\mu}$ is a bounded continuous function and $[\zeta-\omega, \zeta]$ is the fishing zone.

Note that, by extension, $\mu_{p}(t, x, z, C)=0$ if $z \geq \zeta(t, x)$.

Then, the model states that:

1. $a(t, x, z)=u^{+}(t, x)$ if $z<\zeta(t, x)$ and 0 else.

$a$ denotes the horizontal transport of glass eels. It has to be from downstream to upstream. Since the horizontal dynamic of eels is passive, it is possible only when the current is positive.

2. $b(t, x, z)=V_{C} \cdot f_{z}(t, x, z)^{\alpha} \cdot f_{0}(t, x, z)-V_{C} \cdot\left[1-f_{z}(t, x, z)\right]^{\alpha} \cdot\left[1-f_{0}(t, x, z)\right]=b_{\text {down }}(t, x, z)-b_{\text {up }}(t, x, z)$ where $\alpha \geq 1$ is a given value by the experimentation. In practice and in the sequel, $\alpha=1$ is assumed. Then, $b=V_{C} \cdot\left[f_{z}+f_{0}-1\right]$.

$b$ denotes the vertical transport of glass eels. If the current or the light are not favorable, or $z \geq \zeta(t, x)$, glass eels dive to the bottom $\left(b=b_{\text {down }}\right)$. Else, they are able to go towards the surface $\left(b=-b_{\text {up }}\right)$.

3. $d(t, x, z)=H[u(t, x)-\hat{u}] \cdot H\left[\zeta(t, x)-h_{\min }(t, x)-z\right] \cdot k_{v}$.

$d$ denotes the vertical diffusion of glass eels. When the glass eels are able to fight against the current (i.e. $u \geq \hat{u}$ ), and depending on the brightness, they are able to diffuse in the water column.

4. $e(t, x)=H[u(t, x)-\hat{u}] k_{h}$ if $z<\zeta(t, x)$ and 0 else.

$e$ denotes the horizontal diffusion of glass eels. This diffusion is possible as soon as they are able to fight against the current.

Lastly, for any $s \in[0, T]$, let us denote by :

$$
\begin{aligned}
Q & =\{(t, x, z), t \in] 0, T[, x \in] L, R[, z \in] z_{b}(x), z_{\max }[\} \\
\widetilde{Q} & =\{(t, x, z), t \in] 0, T[, x \in] L, R[, z \in] z_{b}(x), \zeta(t, x)[\} \\
\widetilde{\widetilde{Q}} & =\{(t, x, z), t \in] 0, T[, x \in] L, R[, z \in] z_{b}(x), \zeta(t, x)-h_{\min }(t, x)[\}, \\
Q^{+} & =\{(t, x, z) \in Q, u(t, x)>\hat{u}\}, \widetilde{Q}^{+}=Q^{+} \cap \widetilde{Q}, \widetilde{\widetilde{Q}}^{+}=Q^{+} \cap \widetilde{\widetilde{Q}} . \\
\Omega & =\{(x, z), x \in] L, R[, z \in] z_{b}(x), z_{\max }[\}, \\
\widetilde{\Omega}(s) & =\{(x, z) \in \Omega, z<\zeta(s, x)\}, \widetilde{\widetilde{\Omega}}(s)=\left\{(x, z) \in \Omega, z<\zeta(s, x)-h_{\min }(s, x)\right\}, \\
\Omega^{+}(s) & =\{(x, z) \in \Omega, u(s, x)>\hat{u}\}, \widetilde{\Omega}^{+}(s)=\Omega^{+}(s) \cap \widetilde{\Omega}(s), \widetilde{\widetilde{\Omega}}^{+}(s)=\Omega^{+}(s) \cap \widetilde{\widetilde{\Omega}}(s) .
\end{aligned}
$$

We assume that they are all open Lipschitz sets and the corresponding boundaries are: $I=] L, R[$,

$$
\begin{aligned}
\Gamma_{l} & =\left\{\left(x, z_{b}(x)\right), x \in I\right\}, \Gamma^{l}(s)=\{(x, \zeta(s, x)), x \in I\}, \Gamma^{\max }=\left\{\left(x, z_{\max }\right), x \in I\right\} . \\
\Sigma_{l} & =] 0, T\left[\times \Gamma_{l}, \Sigma^{l}=\{(t, x, \zeta(t, x)), t \in] 0, T[, x \in] L, R[\}, \Sigma^{\max }=\right] 0, T\left[\times \Gamma^{\max } .\right. \\
\Gamma_{L} & =\{L\} \times] z_{b}(L), z_{\max }\left[, \widetilde{\Gamma}_{L}(s)=\{L\} \times\right] z_{b}(L), \zeta(s, L)[, \\
\widetilde{\widetilde{\Gamma}}_{L}(s) & =\{L\} \times] z_{b}(L), \zeta(s, L)-h(s, L)\left[, \widetilde{\widetilde{\Gamma}}_{L}^{+}(s)=\widetilde{\widetilde{\Gamma}}_{L}(s) \cap\{u(s, L)>\hat{u}\},\right. \\
\Gamma_{L}^{+}(s) & =\Gamma_{L} \cap\{u(s, L)>\hat{u}\}, \widetilde{\Gamma}_{L}^{+}(s)=\widetilde{\Gamma}_{L}(s) \cap\{u(s, L)>\hat{u}\}, \\
\Gamma_{R} & =\{R\} \times] z_{b}(R), z_{\max }\left[, \widetilde{\Gamma}_{R}(s)=\{R\} \times\right] z_{b}(R), \zeta(s, R)[, \\
\widetilde{\Gamma}_{R}(s) & =\{R\} \times] z_{b}(R), \zeta(s, R)-h(s, R)\left[, \widetilde{\Gamma}_{R}^{+}(s)=\widetilde{\widetilde{\Gamma}}_{R}(s) \cap\{u(s, R)>\hat{u}\},\right. \\
\Gamma_{R}^{+}(s) & =\Gamma_{R} \cap\{u(s, R)>\hat{u}\}, \widetilde{\Gamma}_{R}^{+}(s)=\widetilde{\Gamma}_{R}(s) \cap\{u(s, R)>\hat{u}\}, \\
\Sigma_{R} & =] 0, T\left[\times \Gamma_{R}, \widetilde{\Sigma}_{R}=\cup_{t \in] 0, T[} \widetilde{\Gamma}_{R}(t), \widetilde{\Sigma}_{R}^{+}=\cup_{t \in] 0, T[} \widetilde{\Gamma}_{R}^{+}(t) .\right.
\end{aligned}
$$


Since the natural spatial domain is the river, this domain is a priori $\widetilde{\Omega}(t)$. Thus, it evolves over time and one has to consider the initial/boundary conditions for the domain $\widetilde{Q}$.

Then, the conditions of mixed type are formally the following:

1. the initial condition corresponds to a Dirichlet condition on the boundary $\widetilde{\Omega}(0)$. Assume that no eels are present in the river at time $t=0$, that is $C(t=0)=0$.

2. no eels are able to go out (or in) the domain at the bottom of the river (i.e., $z=z_{b}$ ) and at the surface (i.e., $z=\zeta$ ). Thus, $C \partial_{t} \eta+[\boldsymbol{V} C-D \nabla C] . \eta_{(x, z)}=0$ on $\Sigma_{l}$ and $\Sigma^{l}\left({ }^{2}\right)$.

Note that at the bottom of the river $\left(i . e ., \Sigma_{l}\right), \eta=\frac{\left(0, z_{b}^{\prime},-1\right)}{\sqrt{1+z_{b}^{\prime 2}}}$ and the condition is

$$
[\boldsymbol{V} C-D \nabla C] \cdot \eta_{(x, z)}=0 \quad \text { i.e. } \quad\left(a C-e \partial_{x} C\right) z_{b}^{\prime}+\left(b C+d \partial_{z} C\right)=0
$$

at the surface $\left(i . e ., \Sigma^{l}\right), \eta=\frac{\left(-\partial_{t} \zeta,-\partial_{x} \zeta, 1\right)}{\sqrt{1+\partial_{t} \zeta^{2}+\partial_{x} \zeta^{2}}}$ and the condition is

$$
-C \partial_{t} \zeta-\left(a C-e \partial_{x} C\right) \partial_{x} \zeta-\left(b C+d \partial_{z} C\right)=0 \quad \text { i.e. } \quad-C \partial_{t} \zeta-\left(a C-e \partial_{x} C\right) \partial_{x} \zeta-V_{C} C=0 .
$$

3. One assumes that the diffusion term cancels on $\cup_{t \in] 0, T[} \widetilde{\Gamma}_{R}(t)$. Moreover, when the current is favorable a free outflow convection is possible, else, it cancels. Since $\boldsymbol{V} . \eta=a(t, R, z)=0$ when $u \leq 0$, one gets that $[\boldsymbol{V} C-D \nabla C] . \eta=\boldsymbol{V} . \eta C$. And, in our case, this Fourier-Robin condition becomes $[\boldsymbol{V} C-D \nabla C] . \eta=a C$.

4. On $\cup_{t \in] 0, T[} \widetilde{\Gamma}_{L}(t)$, one assumes that a known density $f(t, z)$ of eels enter the domain. As mentioned in the introduction, when they enter in the domain, glass eels are used to salt water. So, because of the salt wedge, they need to enter in the domain by the lowest part of $\widetilde{\Gamma}_{L}(t)$ and, in practice, $f=0$ unless in a part $] z_{b}(L), z_{b}(L)+\kappa$ [ for a small positive value $\kappa$. Moreover, they enter only when the current allows this entrance. Therefore, the diffusion term cancels and the continuity of the inflow convection gives $\boldsymbol{V} . \eta C=\boldsymbol{V} . \eta f$. Since $\boldsymbol{V} . \eta=-a$, one gets that $[\boldsymbol{V} C-D \nabla C] . \eta=-a f$.

\section{A mathematical formulation}

In this section, we propose a mathematical framework for the study of the model, the definition of a solution and the result of existence.

Although it will not be the best strategy for a numerical approach of this model, it will be more convenient to work in the fixed domain $\Omega$ and not the time-dependent one $\widetilde{\Omega}(t)$ for the mathematical analysis. Then, we propose to extend the equation to Domain $Q=] 0, T[\times \Omega$ by

$$
\left.\partial_{t} C+\operatorname{div}_{(\mathrm{x}, \mathrm{z})}[\boldsymbol{V} C-D \nabla C]=-\mu(., C) C \text { in }\right] 0, T[\times \Omega,
$$

for conditions

$$
\begin{aligned}
& C(t=0)=0 \text { on } \Omega, \quad[\boldsymbol{V} C-D \nabla C] . \eta=0 \text { on } \Sigma_{l}, \quad C=0 \text { on } \Sigma^{\max }, \\
& [\boldsymbol{V} C-D \nabla C] . \eta=a C \text { on }] 0, T\left[\times \Gamma_{R}, \quad[\boldsymbol{V} C-D \nabla C] . \eta=-a f \text { on }\right] 0, T\left[\times \Gamma_{L} .\right.
\end{aligned}
$$

If one denotes by $\tilde{Q}^{c}=Q \backslash c l(\tilde{Q})$, the definitions of $\boldsymbol{V}, D$ and $\mu$ in this part of the domain yield that $C$ has to satisfy in $\tilde{Q}^{c}$ the linear transport equation with constant coefficients

$$
\partial_{t} C-V_{C} \partial_{z} C=0 \quad \text { i.e. } \quad \operatorname{div}_{(\mathrm{t}, \mathrm{x}, \mathrm{z})} C \boldsymbol{U}=0 \quad \text { where } \quad \boldsymbol{U}=\left(1,0,-V_{C}\right)^{T} .
$$

Then, it is well known that there exists a unique (weak) solution as soon as boundary conditions are imposed on the part of the boundary corresponding to $\boldsymbol{U} . \eta<0$ (cf. C. Bardos [6]).

On the one hand, note that $\boldsymbol{U} . \eta=-1$ at $t=0$ and $\boldsymbol{U} . \eta=1$ at $t=T ; \boldsymbol{U} . \eta=0$ at $x=L$ or $x=R$; $\boldsymbol{U} . \eta=-V_{C}$ at $z=z_{\max }$ and $\boldsymbol{U} . \eta=\partial_{t} \zeta+V_{C}$ at $z=\zeta$.

\footnotetext{
${ }^{2} \eta=\left(\partial_{t} \eta, \eta_{(x, z)}\right)$ denotes the outward unit normal to $\widetilde{Q}, \partial_{t} \eta$ is the time component, and $\eta_{(x, z)}$ the spatial one.
} 
Since on the other hand $\partial_{t} \zeta>-V_{C}$, the above extension asserts that $C=0$ in $\tilde{Q}^{c}$ and the compatibility condition in $Q$ across the hyper-surface $z=\zeta$ corresponds to the boundary condition introduced in the model.

Note that this model is of degenerate type. Indeed: in $\widetilde{\widetilde{Q}}^{+}$, the equation is of parabolic type with regular coefficients:

$$
\partial_{t} C-k_{h} \partial_{x x}^{2} C-k_{v} \partial_{z z}^{2} C+\partial_{x}\left(u^{+} C\right)+\partial_{z}\left(b_{u p} C\right)=-\mu_{n} C-\mu_{p}(., C) C
$$

in $\widetilde{Q}^{+} \backslash \widetilde{\widetilde{Q}}^{+}$, it is of degenerate parabolic type, still with regular coefficients, since the second order differentiation exists only for the horizontal direction:

$$
\partial_{t} C-k_{h} \partial_{x x}^{2} C+\partial_{x}\left(u^{+} C\right)-\partial_{z}\left(b_{d o w n} C\right)=-\mu_{n} C ;
$$

in the complementary of $\widetilde{Q}^{+}$, it is of hyperbolic type with regular coefficients:

$$
\partial_{t} C-\partial_{z}\left(b_{\text {down }} C\right)=-\mu_{n} C
$$

Let us set $\boldsymbol{U}:=(C, \boldsymbol{V} C-D \nabla C)$ then the equation writes:

$$
\operatorname{div}_{(\mathrm{t}, \mathrm{x}, \mathrm{z})} \boldsymbol{U}=-\mu(., C) C \text { in the sense of distributions in } Q .
$$

Since one can expect $\mu(., C) C$ to belong to $L^{2}(Q)$, the vector $\boldsymbol{U}$ will belong to the set

$$
H\left(\operatorname{div}_{(\mathrm{t}, \mathrm{x}, \mathrm{z})}, Q\right):=\left\{\boldsymbol{w} \in L^{2}(Q), \operatorname{div}_{(\mathrm{t}, \mathrm{x}, \mathrm{z})} \boldsymbol{w} \in L^{2}(Q)\right\},
$$

endowed with the norm $\boldsymbol{w} \mapsto\left[\|\boldsymbol{w}\|_{L^{2}(Q)}^{2}+\left\|\operatorname{div}_{(\mathrm{t}, \mathrm{x}, \mathrm{z})} \boldsymbol{w}\right\|_{L^{2}(Q)}^{2}\right]^{\frac{1}{2}}$.

Then, by definition of parameters $e$ and $d$, one can expect $C$ to satisfy

$$
\int_{0}^{T} \int_{L}^{R} 1_{\{u>\hat{u}\}}\left(\int_{z_{b}(x)}^{\zeta(t, x)}\left|\partial_{x} C\right|^{2} d z+\int_{z_{b}(x)}^{\zeta(t, x)-h_{\min }(t, x)}\left|\partial_{z} C\right|^{2} d z\right) d x d t<+\infty .
$$

Thus, we will assume that $C$ belongs to the space $\mathcal{W}(0, T)=\left\{v \in L^{2}(Q)\right.$, with $\left.|v|_{\mathcal{W}(0, T)}<+\infty\right\}$ where, for any $v \in \mathcal{W}(0, T),|v|_{\mathcal{W}(0, T)}^{2}:=\int_{0}^{T}\left(\int_{\widetilde{\Omega}^{+}(t)}\left|\partial_{x} v\right|^{2} d x d z+\int_{\widetilde{\Omega}^{+}{ }_{(t)}}\left|\partial_{z} v\right|^{2} d x d z\right) d t$. In the sequel, $\mathcal{W}(0, T)$ will be endowed with the norm $\|v\|_{\mathcal{W}(0, T)}=\left[\|v\|_{L^{2}(Q)}^{2}+|v|_{\mathcal{W}(0, T)}^{2}\right]^{\frac{1}{2}}$.

Moreover, thanks to the equation, it will be proved that if $\mathcal{V}=\left\{v \in H^{1}(\Omega), v=0\right.$ on $\left.\Gamma^{\max }\right\}$, then $\partial_{t} C$ belongs to $L^{2}\left(0, T, \mathcal{V}^{\prime}\right)$.

Therefore, one will have that $C \in C\left([0, T], \mathcal{V}^{\prime}\right)$ (cf. J. L. Lions [13] Vol.7 p.577 sqq.).

Since a classical result for such type of conservation problem leads to $C \in L^{\infty}\left(0, T, L^{2}(\Omega)\right)$, combined with the above continuity, we can consider that $C \in C_{w}\left([0, T], L^{2}(\Omega)\right)^{3}$.

Since $\boldsymbol{U} \in H\left(\operatorname{div}_{(\mathrm{t}, \mathrm{x}, \mathrm{z})}, Q\right)$, it is well known (cf. J. E. Roberts et al. [29]) that the trace of $\boldsymbol{U} . \eta_{(t, x, z)}$ exists in $H^{-\frac{1}{2}}(\partial Q)$ where $\eta_{(t, x, z)}$ denotes the outward unit normal to $\partial Q$. Moreover, for any $v$ in $H^{1}(Q)$, one has the following Green formula:

$$
\int_{Q}\left(\operatorname{div}_{(\mathrm{t}, \mathrm{x}, \mathrm{z})} \boldsymbol{U}\right) v d x d z d t+\int_{Q} \boldsymbol{U} \cdot\left(\partial_{t} v, \nabla v\right) d x d z d t=<\boldsymbol{U} \cdot \eta_{(t, x, z)}, v>_{H^{-\frac{1}{2}}(\partial Q), H^{\frac{1}{2}}(\partial Q)} .
$$

\footnotetext{
${ }^{3}$ The continuous functions from $[0, T]$ to $L^{2}(\Omega)$ endowed with the weak topology (cf. J. L. Lions et al.) [24].
} 
Then, the formal boundary conditions imply that $U . \eta$ is a function such that

$$
\boldsymbol{U} \cdot \eta_{(t, x, z)}=(C, C \boldsymbol{V}-D \nabla C) \cdot \eta_{(t, x, z)}= \begin{cases}-C(t=0)=0 & \text { in } \Omega \\ 0 & \text { on } z=z_{b} \text { or } z=z_{\max } \\ a C & \text { on } x=R \\ -a f & \text { on } x=L\end{cases}
$$

Thanks to this remark, one can expect looking for $(C, \boldsymbol{V} C-D \nabla C)$ in the set

$$
\mathcal{H}\left(\operatorname{div}_{(\mathrm{t}, \mathrm{x}, \mathrm{z})}, Q\right):=\left\{\boldsymbol{w} \in H\left(\operatorname{div}_{(\mathrm{t}, \mathrm{x}, \mathrm{z})}, Q\right), \boldsymbol{w} \cdot \eta_{(t, x, z)} \in L^{2}(\partial Q)\right\},
$$

endowed with the norm $\boldsymbol{w} \mapsto\left[\|\boldsymbol{w}\|_{H\left(\operatorname{div}_{(\mathrm{t}, \mathrm{x}, \mathrm{z})}, Q\right)}^{2}+\left\|\boldsymbol{w} \cdot \eta_{(t, x, z)}\right\|_{L^{2}(\partial Q)}^{2}\right]^{\frac{1}{2}}$.

For that, we need to precise the sense given to the trace of $a C$ on $\Gamma_{R}$.

Since $a=0$ if $u \leq \hat{u}$, the support of $a(t,$.$) on \Gamma_{R}$ is contained in $\widetilde{\Gamma}_{R}(t)$ which is a part of the vertical boundary of $\widetilde{\Omega}^{+}(t)$. Then, $C$ has a trace in $L^{2}\left(\widetilde{\Sigma}_{R}^{+}\right)$with the estimation

$$
\int_{0}^{T} \int_{z_{b}(R)}^{\zeta(t, R)} 1_{\{u(t, R)>\hat{u}\}}|C(t, R, z)|^{2} d z d t \leq c \int_{0}^{T} \int_{L}^{R} \int_{z_{b}(x)}^{\zeta(t, x)} 1_{\{u(t, x)>\hat{u}\}}\left[|C|^{2}+\left|\partial_{x} C\right|^{2}\right] d z d x d t
$$

where $c$ is a constant depending on $R-L$, the horizontal size of the domain.

Seeing $a$ as a weight, one gives a sense to $a C$ in $L^{2}\left(\Sigma_{R}\right)$ with a control of its norm by the one of $C$ in $\mathcal{W}(0, T)$.

For a reason of convenience in the proofs, denote by $\lambda>\lambda_{1}$ a constant where $\lambda_{1}$ is defined in Lemma $4.2^{4}$. Then, if one denotes by $S=e^{-\lambda t} C$,

$$
\partial_{t} S+\lambda S-\operatorname{div}_{(\mathrm{x}, \mathrm{z})}[D \nabla S]+\operatorname{div}_{(\mathrm{x}, \mathrm{z})}(\boldsymbol{V} S)=-\mu\left(., e^{\lambda t} S\right) S=-\bar{\mu}(., S) S .
$$

Thus, denoting by $\bar{f}=e^{-\lambda t} f$, the result we will be interested in is

Theorem 3.1. There exists nonnegative $S$ in $\mathcal{W}(0, T) \cap C_{w}\left(0, T, L^{2}(\Omega)\right)$ such that $\partial_{t} S \in L^{2}\left(0, T, \mathcal{V}^{\prime}\right)$ and $S(t=0)=0$, solution for a.e. $t \in] 0, T[$, to the variational problem: for any $v$ in $\mathcal{V}$,

$$
<\partial_{t} S, v>+\int_{\Omega}\left[(\nabla S)^{T} . D \cdot \nabla v-S \boldsymbol{V} \cdot \nabla v+\lambda S v+\bar{\mu}(., S) S v\right] d x d z+\int_{\Gamma_{R}} a S v d \sigma=\int_{\Gamma_{L}} a \bar{f} v d \sigma .
$$

Then, $C$ is given by the relation: $C=e^{\lambda t} S$.

\section{Existence of a solution}

In this section, we prove the above theorem by using a method of vanishing diffusion.

The control of the norms of the approximate solution, for the a priori estimates, comes from the remark that the lack of information in the hyperbolic part will concentrate, thanks to a by part-integration, on a part of the boundary $\left\{z=\zeta-h_{\min }\right\}$. Then, since it will be a part of the boundary of $\widetilde{\widetilde{Q}}^{+}$, the parabolic part of the problem, one will use it as a trace term.

For the nonlinear term, $\bar{\mu}$, we will use the compactness information coming from the same parabolic part of the problem.

For any positive $\epsilon$ and any $S_{\epsilon}, v \in \mathcal{V}$, denote by

$$
\mathcal{A}_{\epsilon}:\left(S_{\epsilon}, v\right) \mapsto \int_{\Omega}\left[\left(\nabla S_{\epsilon}\right)^{T} \cdot D \cdot \nabla v+\epsilon \nabla S_{\epsilon} \cdot \nabla v-S_{\epsilon} \boldsymbol{V} \cdot \nabla v+\lambda S_{\epsilon} v\right] d x d z+\int_{\Gamma_{R}} a S_{\epsilon} v d \sigma .
$$

\footnotetext{
${ }^{4}$ It will be useful in the sequel to obtain a priori estimates.
} 
Lemma 4.1. If $u \in H^{1}(A)$ for a regular domain $A$, for any Borel-function $g: \mathbb{R} \rightarrow \mathbb{R}$ with $|g(x)| \leq$ $\beta|x|+\gamma$ for nonnegative $\beta$ and $\gamma$, then $g(u) \partial_{x_{i}} u=\partial_{x_{i}} \int_{0}^{u} g(s) d s$ and $u \partial_{x_{i}} g(u)=\partial_{x_{i}} \int_{0}^{u} s g^{\prime}(s) d s$ if $g$ is Lipschitz-continuous.

Proof. On the one hand, thanks to the theorem of Lebesgue and the chain rule in the Sobolev spaces, denoting by $g_{M}(s)=\max [-M, \min (M, g(s))]$, one has, in $L^{1}(A)$, that

$$
g(u) \partial_{x_{i}} u=\lim _{M} g_{M}(u) \partial_{x_{i}} u=\lim _{M} \partial_{x_{i}} \int_{0}^{u} g_{M}(s) d s
$$

On the other hand, when $M$ goes to infinity, for any real $x$,

$$
\left|\int_{0}^{x} g_{M}(s) d s-\int_{0}^{x} g(s) d s\right| \leq \int_{-|x|}^{|x|}\left|g_{M}(s)-g(s)\right| d s \leq \int_{-|x|}^{|x|}(|g(s)|-M)^{+} d s \rightarrow 0 ;
$$

and

$$
\left|\int_{0}^{u} g_{M}(s) d s\right| \leq \int_{-|u|}^{|u|}\left|g_{M}(s)\right| d s \leq \int_{-|u|}^{|u|}|g(s)| d s \leq \int_{-|u|}^{|u|} \beta|s|+\gamma d s \leq \beta u^{2}+2 \gamma|u| .
$$

Thus, thanks to the dominated convergence theorem, $\int_{0}^{u} g_{M}(s) d s$ converges to $\int_{0}^{u} g(s) d s$ in $L^{1}(A)$ and $\partial_{x_{i}} \int_{0}^{u} g_{M}(s) d s$ converges to $\partial_{x_{i}} \int_{0}^{u} g(s) d s$ in the sense of distributions in $A$ and the first result holds.

The second part is a corollary of the first one since $u \partial_{x_{i}} g(u)=g^{\prime}(u) u \partial_{x_{i}} u$ where $x \mapsto g^{\prime}(x) x$ satisfies the assumption of the first part when $g$ is a Lipschitz-continuous function.

Lemma 4.2. Assume that $\lambda \geq \lambda_{1}=\frac{\|u\|_{\infty}^{2}}{k_{h}}+3 \frac{V_{C}^{2}}{k_{v}}+2\left[V_{C}+\frac{V_{C}}{\bar{h}}\right]+1$, then, for any nondecreasing Lipschitzcontinuous function $\psi$ with $\psi(0)=0$,

$$
\begin{aligned}
\mathcal{A}_{\epsilon}\left(S_{\epsilon}, \psi\left(S_{\epsilon}\right)\right) \geq & \left.\int_{\Omega} \psi^{\prime}\left(S_{\epsilon}\right)\left[\left(\frac{e}{4}+\epsilon\right)\left|\partial_{x} S_{\epsilon}\right|^{2}+\left(\frac{d}{4}+\epsilon\right)\left|\partial_{z} S_{\epsilon}\right|^{2}\right)\right] d x d z \\
& +\int_{\Omega}\left[\lambda S_{\epsilon} \psi\left(S_{\epsilon}\right)-\frac{\lambda-1}{2}\left(\psi^{\prime}\left(S_{\epsilon}\right) S_{\epsilon}^{2}+\left|G\left(S_{\epsilon}\right)\right|\right)\right] d x d z+\int_{\Gamma_{R}} a S_{\epsilon} \psi\left(S_{\epsilon}\right) d \sigma
\end{aligned}
$$

where $G(x)=\int_{0}^{x} \psi^{\prime}(t) t d t$.

Proof. Thanks to the chain rule in the Sobolev spaces, $v=\psi\left(S_{\epsilon}\right) \in \mathcal{V}$ and

$$
\begin{aligned}
\mathcal{A}_{\epsilon}\left(S_{\epsilon}, \psi\left(S_{\epsilon}\right)\right)= & \int_{\Omega}\left[\psi^{\prime}\left(S_{\epsilon}\right)\left(\nabla S_{\epsilon}\right)^{T} \cdot D \cdot \nabla S_{\epsilon}-\psi^{\prime}\left(S_{\epsilon}\right) S_{\epsilon} \boldsymbol{V} \cdot \nabla S_{\epsilon}+\lambda S_{\epsilon} \psi\left(S_{\epsilon}\right)\right] d x d z \\
& +\epsilon \int_{\Omega} \psi^{\prime}\left(S_{\epsilon}\right)\left|\nabla S_{\epsilon}\right|^{2} d x d z+\int_{\Gamma_{R}} a S_{\epsilon} \psi\left(S_{\epsilon}\right) d \sigma .
\end{aligned}
$$

I.e., one gets that

$$
\begin{aligned}
\mathcal{A}_{\epsilon}\left(S_{\epsilon}, \psi\left(S_{\epsilon}\right)\right)= & \int_{\Omega}\left[\psi^{\prime}\left(S_{\epsilon}\right)\left(e\left|\partial_{x} S_{\epsilon}\right|^{2}+d\left|\partial_{z} S_{\epsilon}\right|^{2}\right)-\psi^{\prime}\left(S_{\epsilon}\right) S_{\epsilon}\left(a \partial_{x} S_{\epsilon}-b \partial_{z} S_{\epsilon}\right)\right] d x d z \\
& +\int_{\Omega}\left[\epsilon \psi^{\prime}\left(S_{\epsilon}\right)\left|\nabla S_{\epsilon}\right|^{2}+\lambda S_{\epsilon} \psi\left(S_{\epsilon}\right)\right] d x d z+\int_{\Gamma_{R}} a S_{\epsilon} \psi\left(S_{\epsilon}\right) d \sigma .
\end{aligned}
$$

Note that,

If $e=0$ then $a=0$, otherwise $e=k_{h}$ and $a=u^{+}$.

If $d=0$ then $b_{u p}=0$, otherwise $d=k_{v}$ and $b_{u p}=V_{C} \cdot\left[1-f_{z}(t, x, z)\right] \leq V_{C}$. 
Thus, for positive $\beta$ and $\gamma$, Young's inequality yields

$$
\begin{aligned}
& e\left|\partial_{x} S_{\epsilon}\right|^{2}+d\left|\partial_{z} S_{\epsilon}\right|^{2}-S_{\epsilon}\left(a \partial_{x} S_{\epsilon}-b \partial_{z} S_{\epsilon}\right) \\
\geq & \left(e-\beta a^{2}\right)\left|\partial_{x} S_{\epsilon}\right|^{2}+\left(d-\gamma b_{u p}^{2}\right)\left|\partial_{z} S_{\epsilon}\right|^{2}-S_{\epsilon}^{2}\left(\frac{1}{4 \beta}+\frac{1}{4 \gamma}\right)+b_{\text {down }} S_{\epsilon} \partial_{z} S_{\epsilon} \\
\geq & \left(1-\beta \frac{\|u\|_{\infty}^{2}}{k_{h}}\right) e\left|\partial_{x} S_{\epsilon}\right|^{2}+\left(1-\gamma \frac{V_{C}^{2}}{k_{v}}\right) d\left|\partial_{z} S_{\epsilon}\right|^{2}-S_{\epsilon}^{2}\left(\frac{1}{4 \beta}+\frac{1}{4 \gamma}\right)+b_{\text {down }} S_{\epsilon} \partial_{z} S_{\epsilon} \\
\geq & \frac{e}{2}\left|\partial_{x} S_{\epsilon}\right|^{2}+\frac{d}{2}\left|\partial_{z} S_{\epsilon}\right|^{2}-S_{\epsilon}^{2}\left(\frac{\|u\|_{\infty}^{2}}{2 k_{h}}+\frac{V_{C}^{2}}{2 k_{v}}\right)+b_{\text {down }} S_{\epsilon} \partial_{z} S_{\epsilon},
\end{aligned}
$$

if $\beta=\frac{k_{h}}{2\|u\|_{\infty}^{2}}$ and $\gamma=\frac{k_{v}}{2 V_{C}^{2}}$. Then, thanks to Lemma 4.1,

$$
\begin{aligned}
\mathcal{A}_{\epsilon}\left(S_{\epsilon}, \psi\left(S_{\epsilon}\right)\right) & \geq \int_{\Omega}\left[\lambda S_{\epsilon} \psi\left(S_{\epsilon}\right)-\psi^{\prime}\left(S_{\epsilon}\right) \frac{S_{\epsilon}^{2}}{2}\left(\frac{\|u\|_{\infty}^{2}}{k_{h}}+\frac{V_{C}^{2}}{k_{v}}\right)\right] d x d z+\int_{\Gamma_{R}} a S_{\epsilon} \psi\left(S_{\epsilon}\right) d \sigma \\
+ & \int_{\Omega}\left[\epsilon \psi^{\prime}\left(S_{\epsilon}\right)\left|\nabla S_{\epsilon}\right|^{2}+\frac{\psi^{\prime}\left(S_{\epsilon}\right)}{2}\left(e\left|\partial_{x} S_{\epsilon}\right|^{2}+d\left|\partial_{z} S_{\epsilon}\right|^{2}\right)+b_{d o w n} \partial_{z} \int_{0}^{S_{\epsilon}} \psi^{\prime}(\tau) \tau d \tau\right] d x d z
\end{aligned}
$$

But,

$$
\begin{aligned}
& \int_{\Omega} b_{\text {down }} \partial_{z} \int_{0}^{S_{\epsilon}}\left[\psi^{\prime}(\tau) \tau\right] d \tau d x d z \\
= & \int_{L}^{R} 1_{\{u(t, x) \geq \hat{u}\}} \int_{\zeta(t, x)-h_{\min }(t, x)}^{z_{\max }} V_{C} \min \left[1, \frac{\left(z-z_{b}(x)\right)^{+}}{\zeta(t, x)-z_{b}(x)}\right] \partial_{z} \int_{0}^{S_{\epsilon}}\left[\psi^{\prime}(\tau) \tau\right] d \tau d z d x \\
& +\int_{L}^{R} 1_{\{u(t, x)<\hat{u}\}} \int_{Z_{b}}^{z_{\max }} V_{C} \min \left[1, \frac{\left(z-z_{b}(x)\right)^{+}}{\zeta(t, x)-z_{b}(x)}\right] \partial_{z} \int_{0}^{S_{\epsilon}}\left[\psi^{\prime}(\tau) \tau\right] d \tau d z d x \\
= & \int_{L}^{R} 1_{\{u(t, x) \geq \hat{u}\}} V_{C} \int_{0}^{S_{\epsilon}\left(t, x, z_{\max }\right)}\left[\psi^{\prime}(\tau) \tau\right] d \tau d x \quad\left(=0 \operatorname{since} S_{\epsilon}\left(t, x, z_{\max }\right)=0\right) \\
& +\int_{L}^{R} 1_{\{u(t, x)<\hat{u}\}} V_{C} \int_{0}^{S_{\epsilon}\left(t, x, z_{\max }\right)}\left[\psi^{\prime}(\tau) \tau\right] d \tau d x \quad\left(=0 \operatorname{since} S_{\epsilon}\left(t, x, z_{\max }\right)=0\right) \\
& -\int_{L}^{R} 1_{\{u(t, x) \geq \hat{u}\}} V_{C}\left[\frac{\zeta(t, x)-h_{\min }(t, x)-z_{b}(x)}{\zeta(t, x)-z_{b}(x)}\right] \int_{0}^{S_{\epsilon}\left(t, x, \zeta(t, x)-h_{\min }(t, x)\right)}\left[\psi^{\prime}(\tau) \tau\right] d \tau d x \\
& -\int_{L}^{R} 1_{\{u(t, x) \geq \hat{u}\}} \int_{\zeta(t, x)-h_{\min }(t, x)}^{\zeta(t, x)}\left[\frac{V_{C}}{\zeta(t, x)-z_{b}(x)}\right] \int_{0}^{S_{\epsilon}}\left[\psi^{\prime}(\tau) \tau\right] d \tau d z d x \\
& -\int_{L}^{R} 1_{\{u(t, x)<\hat{u}\}} \int_{Z_{b}(x)}^{\zeta(t, x)}\left[\frac{V_{C}}{\zeta(t, x)-z_{b}(x)}\right] \int_{0}^{S_{\epsilon}}\left[\psi^{\prime}(\tau) \tau\right] d \tau d z d x .
\end{aligned}
$$

Then,

$$
\begin{aligned}
\int_{\Omega} b_{\text {down }} \partial_{z} \int_{0}^{S_{\epsilon}}\left[\psi^{\prime}(\tau) \tau\right] d \tau d x d z \geq & -\int_{L}^{R} 1_{\{u(t, x) \geq \hat{u}\}} V_{C}\left|\int_{0}^{S_{\epsilon}\left(t, x, \zeta(t, x)-h_{\min }(t, x)\right)}\left[\psi^{\prime}(\tau) \tau\right] d \tau\right| d x \\
& -\int_{\Omega}\left[\frac{V_{C}}{\zeta(t, x)-z_{b}(x)}\right]\left|\int_{0}^{S_{\epsilon}}\left[\psi^{\prime}(\tau) \tau\right] d \tau\right| d x d z \\
\geq & -\int_{L}^{R} 1_{\{u(t, x) \geq \hat{u}\}} V_{C} G\left[S_{\epsilon}\left(t, x, \zeta(t, x)-h_{\min }(t, x)\right)\right] d x \\
& -\int_{\Omega}\left[\frac{V_{C}}{\zeta(t, x)-z_{b}(x)}\right] G\left(S_{\epsilon}\right) d x d z .
\end{aligned}
$$


For any $z \in] z_{b}(x), \zeta(t, x)-h_{\min }(t, x)[$,

$$
\begin{aligned}
& \left|G\left[S_{\epsilon}\left(t, x, \zeta(t, x)-h_{\min }(t, x)\right)\right]\right| \\
\leq & \left|G\left[S_{\epsilon}(t, x, z)\right]\right|+\left|\int_{z_{b}(x)}^{\zeta(t, x)-h_{\min }(t, x)} \partial_{z} G\left[S_{\epsilon}(t, x, \sigma)\right] d \sigma\right| \\
\leq & \left|G\left[S_{\epsilon}(t, x, z)\right]\right|+\left|\int_{z_{b}(x)}^{\zeta(t, x)-h_{\min }(t, x)} \psi^{\prime}\left(S_{\epsilon}(t, x, \sigma)\right) S_{\epsilon}(t, x, \sigma) \partial_{z} S_{\epsilon}(t, x, \sigma) d \sigma\right| \\
\leq & \left|G\left[S_{\epsilon}(t, x, z)\right]\right|+\alpha \int_{z_{b}(x)}^{\zeta(t, x)-h_{\min }(t, x)} \psi^{\prime}\left(S_{\epsilon}(t, x, \sigma)\right)\left|\partial_{z} S_{\epsilon}(t, x, \sigma)\right|^{2} d \sigma \\
& +\frac{1}{4 \alpha} \int_{z_{b}(x)}^{\zeta(t, x)-h_{\min }(t, x)} \psi^{\prime}\left(S_{\epsilon}(t, x, \sigma)\right)\left|S_{\epsilon}(t, x, \sigma)\right|^{2} d \sigma
\end{aligned}
$$

and,

$$
\begin{aligned}
& \int_{L}^{R} 1_{\{u(t, x) \geq \hat{u}\}} V_{C} G\left[S_{\epsilon}\left(t, x, \zeta(t, x)-h_{\min }(t, x)\right)\right] d x \\
\leq & V_{C} \int_{L}^{R} 1_{\{u(t, x) \geq \hat{u}\}}\left|G\left[S_{\epsilon}(t, x, z)\right]\right| d x \\
& +V_{C} \alpha \int_{L}^{R} 1_{\{u(t, x) \geq \hat{u}\}} \int_{z_{b}(x)}^{\zeta(t, x)-h_{\min }(t, x)} \psi^{\prime}\left(S_{\epsilon}(t, x, \sigma)\right)\left|\partial_{z} S_{\epsilon}(t, x, \sigma)\right|^{2} d \sigma d x \\
& +\frac{V_{C}}{4 \alpha} \int_{L}^{R} 1_{\{u(t, x) \geq \hat{u}\}} \int_{z_{b}(x)}^{\zeta(t, x)-h_{\min }(t, x)} \psi^{\prime}\left(S_{\epsilon}(t, x, \sigma)\right)\left|S_{\epsilon}(t, x, \sigma)\right|^{2} d \sigma d x
\end{aligned}
$$

From (4.2), if $\alpha=\frac{k_{v}}{4 V_{C}}$,

$$
\begin{aligned}
& \mathcal{A}_{\epsilon}\left(S_{\epsilon}, \psi\left(S_{\epsilon}\right)\right) \\
\geq & \int_{\Omega}\left[\epsilon \psi^{\prime}\left(S_{\epsilon}\right)\left|\nabla S_{\epsilon}\right|^{2}+\frac{\psi^{\prime}\left(S_{\epsilon}\right)}{4}\left(e\left|\partial_{x} S_{\epsilon}\right|^{2}+d\left|\partial_{z} S_{\epsilon}\right|^{2}\right)\right] d x d z+\int_{\Gamma_{R}} a S_{\epsilon} \psi\left(S_{\epsilon}\right) d \sigma \\
& \left.+\int_{\Omega}\left[\lambda S_{\epsilon} \psi\left(S_{\epsilon}\right)-\psi^{\prime}\left(S_{\epsilon}\right) \frac{S_{\epsilon}^{2}}{2}\left(\frac{\|u\|_{\infty}^{2}}{k_{h}}+3 \frac{V_{C}^{2}}{k_{v}}\right)-\left[V_{C}+\frac{V_{C}}{\bar{h}}\right] \mid G\left(S_{\epsilon}\right)\right]\right] d x d z
\end{aligned}
$$

and the result holds by assumption on $\lambda$.

Proposition 4.3. Assume that $\lambda>\lambda_{1}$ and that $\epsilon>0$. Then, there exists an element $S_{\epsilon}$ in $L^{2}(0, T, \mathcal{V})$ such that $\partial_{t} S_{\epsilon} \in L^{2}\left(0, T, \mathcal{V}^{\prime}\right)$ and $S_{\epsilon}(t=0)=0$, solution, $\left.t \in\right] 0, T[$ a.e., to the variational problem: for any $v$ in $\mathcal{V}$,

$$
\begin{aligned}
& <\partial_{t} S_{\epsilon}, v>+\int_{\Omega}\left[\epsilon \nabla S_{\epsilon} \cdot \nabla v+\left(\nabla S_{\epsilon}\right)^{T} \cdot D \cdot \nabla v-S_{\epsilon} \boldsymbol{V} \cdot \nabla v+\lambda S_{\epsilon} v+\bar{\mu}\left(., S_{\epsilon}\right) S_{\epsilon} v\right] d x d z \\
& +\int_{\Gamma_{R}} a S_{\epsilon} v d \sigma=\int_{\Gamma_{L}} a \bar{f} v d \sigma .
\end{aligned}
$$

Proof. The bilinear form $\mathcal{A}_{\epsilon}$ is continuous and coercive on $\mathcal{V} \times \mathcal{V}$ thanks to Lemma 4.2 when considering $\psi=I d$. Indeed,

$$
\mathcal{A}_{\epsilon}\left(S_{\epsilon}, S_{\epsilon}\right) \geq \int_{\Omega}\left[\left(\frac{e}{4}+\epsilon\right)\left|\partial_{x} S_{\epsilon}\right|^{2}+\left(\frac{d}{4}+\epsilon\right)\left|\partial_{z} S_{\epsilon}\right|^{2}+S_{\epsilon}^{2}\right] d x d z+\int_{\Gamma_{R}} a S_{\epsilon}^{2} d \sigma d t .
$$

Then, since $\bar{\mu}$ is a nonnegative, bounded continuous function, the result comes from classical result on linear parabolic equations and a standard application of the fixed-point theorem of Schauder (cf. A. Ambrosetti et al. [3] p.43 or G. Gagneux et al. [17] p.29 sqq. for exemple). 
Proposition 4.4. $S_{\epsilon}$ is a nonnegative function such that, independently of $\epsilon, S_{\epsilon}$ is bounded in $\mathcal{W}(0, T)$ and in $L^{\infty}\left(0, T, L^{2}(\Omega)\right)$ and $\sqrt{\epsilon} S_{\epsilon}$ is bounded in $L^{2}\left(0 ; T, H^{1}(\Omega)\right)$.

Proof. Consider Lemma 4.2 with $\psi(x)=-x^{-}=-(-x)^{+}$. Then, the time integration yields

$$
\int_{\Omega}\left(S_{\epsilon}^{-}\right)^{2}(T) d x d z+\int_{Q} \frac{1}{4}\left(e\left|\partial_{x} S_{\epsilon}^{-}\right|^{2}+d\left|\partial_{z} S_{\epsilon}^{-}\right|^{2}\right)+\left(S_{\epsilon}^{-}\right)^{2} d x d z d t+\int_{0}^{T} \int_{\Gamma_{R}} a\left(S_{\epsilon}^{-}\right)^{2} d \sigma d t \leq 0 .
$$

Thus, $S_{\epsilon}^{-}=0$ and $S_{\epsilon} \geq 0$.

We propose a second proof of the same result, in the idea of D. Gilbarg et al. [20] p.192 sqq. or J. Droniou [16]. The interest of this second one is that it can be adapted to more general situations (with the only $L^{\infty}$ information on $\left.\boldsymbol{V}\right)$. For any positive $\delta$, consider $\psi(x)=-\frac{x+\delta}{x} 1_{\{x<-\delta\}}$ and $\Psi(x)=\int_{0}^{x} \psi(s) d s$, to get:

$$
\begin{aligned}
& \int_{Q}\left[\partial_{t} \Psi\left(S_{\epsilon}\right)+\epsilon \psi^{\prime}\left(S_{\epsilon}\right)\left|\nabla S_{\epsilon}\right|^{2}-\psi^{\prime}\left(S_{\epsilon}\right) S_{\epsilon} \boldsymbol{V} . \nabla S_{\epsilon}\right] d x d z d t \leq 0, \\
& \text { i.e. } \\
& \int_{\Omega} \Psi\left(S_{\epsilon}(T)\right) d x d z+\epsilon \delta \int_{Q \cap\left\{S_{\epsilon}<-\delta\right\}} \frac{\left|\nabla S_{\epsilon}\right|^{2}}{S_{\epsilon}^{2}} d t d x d z \leq \delta \max \left(V_{C},\|u\|_{\infty}\right) \int_{Q \cap\left\{S_{\epsilon}<-\delta\right\}} \frac{\left|\nabla S_{\epsilon}\right|}{\left|S_{\epsilon}\right|} d x d z d t, \\
& \text { and } \int_{Q \cap\left\{S_{\epsilon}<-\delta\right\}} \frac{\left|\nabla S_{\epsilon}\right|^{2}}{S_{\epsilon}^{2}} d t d x d z \leq C t e<+\infty .
\end{aligned}
$$

If one denotes $F_{\delta}(x)=\ln \left[1+\frac{(-x-\delta)^{+}}{\delta}\right]$, this yields,

$$
\int_{Q}\left|\nabla F_{\delta}\left(S_{\epsilon}\right)\right|^{2} d t d x d z \leq C t e<+\infty
$$

and, thanks to the inequality of Poincaré in $\mathcal{V}$,

$$
\int_{Q}\left|\ln \left[1+\frac{\left(-S_{\epsilon}-\delta\right)^{+}}{\delta}\right]\right|^{2} d t d x d z \leq C t e<+\infty
$$

Then, passing to the limit over $\delta$ to $0^{+}$, Beppo Levi theorem ensures $S_{\epsilon} \geq 0$.

Concerning the a priori estimates, thanks to Inequality (4.4), one has, for any positive $\alpha$ and any $t$, that

$$
\begin{aligned}
& \frac{1}{2}\left\|S_{\epsilon}(t)\right\|_{L^{2}(\Omega)}^{2}+\int_{0}^{t} \int_{\Omega}\left[\left(\frac{e}{4}+\epsilon\right)\left|\partial_{x} S_{\epsilon}\right|^{2}+\left(\frac{d}{4}+\epsilon\right)\left|\partial_{z} S_{\epsilon}\right|^{2}+S_{\epsilon}^{2}\right] d x d z d s+\int_{0}^{t} \int_{\Gamma_{R}} a S_{\epsilon}^{2} d \sigma d s \\
& \leq \frac{1}{4 \alpha} \int_{0}^{t} \int_{\Gamma_{L}} a f^{2} d \sigma d s+\alpha \int_{0}^{t} \int_{\Gamma_{L}} a S_{\epsilon}^{2} d \sigma d s \leq \frac{C(f)}{\alpha}+\alpha \int_{0}^{t} \int_{\widetilde{\Gamma}_{L}^{+}(s)} a S_{\epsilon}^{2} d \sigma d s .
\end{aligned}
$$

Since $\widetilde{\Gamma}_{L}^{+}(s)$ is a part of the vertical boundary of $\widetilde{\Omega}^{+}(s)$, there exists a positive constant $c$, depending on $R-L$, such that

$$
\frac{1}{2}\left\|S_{\epsilon}(t)\right\|_{L^{2}(\Omega)}^{2}+\frac{1}{4}\left\|S_{\epsilon}\right\|_{\mathcal{W}(0, t)}^{2}+\epsilon \int_{0}^{t} \int_{\Omega}\left|\nabla S_{\epsilon}\right|^{2} d x d z d s+\int_{0}^{t} \int_{\Gamma_{R}} a S_{\epsilon}^{2} d \sigma d s \leq \frac{C(f)}{\alpha}+c \alpha\left\|S_{\epsilon}\right\|_{\mathcal{W}(0, t)}^{2} .
$$

Then the proposition is proved if $\alpha=\frac{1}{8 c}$.

Remark 4.5. Since we are in a bounded domain, the boundedness in $L^{2}$ implies the control of the $L^{1}$ norm, i.e. the total mass of the population since $S_{\epsilon} \geq 0$. 
Note that this control could also be obtained by using $\psi(x)=\max (-1, \min (n x, 1))$ in Lemma 4.2. Then, passing to the limit when $n$ goes to $\infty$ ensures

$$
\int_{\Omega}\left|S_{\epsilon}\right|(T) d x d z+\lambda \int_{Q}\left|S_{\epsilon}\right| d x d z d t+\int_{0}^{T} \int_{\Gamma_{R}} a\left|S_{\epsilon}\right| d \sigma d t \leq \int_{0}^{T} \int_{\Gamma_{L}} a|\bar{f}| d \sigma d t .
$$

On the other hand, a control of the $L^{\infty}$-norm seems out of range. Indeed, when the migration is not possible, the model yields an aggregation of the population at the bottom of the domain.

Let us mention that, similarly, one has that $\mathcal{S}_{\epsilon}: u_{0} \mapsto S_{\epsilon}$ is a semigroup of contraction in $L^{1}$.

As a consequence of the previous proposition, one has that

Corollary 4.6. Independently of $\epsilon, \partial_{t} S_{\epsilon}$ is bounded in $L^{2}\left(0, T, \mathcal{V}^{\prime}\right)$.

Then, a first consequence of these results is that

Lemma 4.7. There exist a nonnegative $\chi \in L^{2}(Q)$ and $S$ in $\mathcal{W}(0, T) \cap C_{w}\left(0, T, L^{2}(\Omega)\right)$ such that $\partial_{t} S \in$ $L^{2}\left(0, T, \mathcal{V}^{\prime}\right)$ and $S(t=0)=0$, solution for a.e. $\left.t \in\right] 0, T$, to the variational problem: for any $v$ in $\mathcal{V}$,

$$
<\partial_{t} S, v>+\int_{\Omega}\left[(\nabla S)^{T} \cdot D \cdot \nabla v-S \boldsymbol{V} \cdot \nabla v+\lambda S v+\chi v\right] d x d z+\int_{\Gamma_{R}} a S v d \sigma=\int_{\Gamma_{L}} a \bar{f} v d \sigma .
$$

Proof. This result is a consequence of the a priori estimates and since $\bar{\mu}\left(., S_{\epsilon}\right) S_{\epsilon}$ is bounded in $L^{2}(Q)$.

To complete the result of existence, a compactness argument is needed to identify $\chi$.

Consider $0 \leq t_{0}<t_{1} \leq T, A \subset \Omega$ a regular nonempty domain such that

]$t_{0}, t_{1}\left[\times A \subset\left\{(t, x, z) \in Q, u(t, x)>0, z_{b}(x)<z<\zeta(t, x)-h_{\min }(t, x)\right\}\right.$ and $\varphi$ be a nonnegative fixed function in $\mathcal{D}(Q)$ such that $\operatorname{supp} \varphi \subset\left[t_{0}, t_{1}\right] \times A$.

On the one hand, since $S_{\epsilon} \in L^{2}\left(t_{0}, t_{1}, H^{1}(A)\right)$, one gets that $S_{\epsilon} \varphi \in L^{2}\left(t_{0}, t_{1}, H_{0}^{1}(A)\right)$, and the norm is bounded independently of $\epsilon$ thanks to the a priori estimates.

For any $v \in H^{1}(A)$, one has that $\varphi v \in H_{0}^{1}(A)$. It is a test function and one gets

$$
\begin{aligned}
& <\partial_{t} S_{\epsilon}, \varphi v> \\
& \quad+\int_{\Omega}\left(\nabla S_{\epsilon}\right)^{T} \cdot D \cdot \nabla[\varphi v]-S_{\epsilon} \boldsymbol{V} \cdot \nabla[\varphi v]+\lambda S_{\epsilon} \varphi v+\bar{\mu}\left(., S_{\epsilon}\right) S_{\epsilon} \varphi v+\epsilon \nabla S_{\epsilon} \cdot \nabla \varphi v d x d z=0, \\
& \quad \quad \text { i.e. } \\
& \left|<\partial_{t} S_{\epsilon}, \varphi v>\right| \leq\left[c(D, \varphi)\left\|\nabla S_{\epsilon}\right\|_{L^{2}(A)}+c(\boldsymbol{V}, \varphi, \lambda, \mu)\left\|S_{\epsilon}\right\|_{L^{2}(A)}+\epsilon c(\varphi)\left\|\nabla S_{\epsilon}\right\|_{L^{2}(A)}\right]\|v\|_{H^{1}(A)}
\end{aligned}
$$

and since $\varphi$ is a fixed function in $\mathcal{D}(Q)$,

$$
\left|<\partial_{t}\left(\varphi S_{\epsilon}\right), v>\right| \leq\left|<\partial_{t} S_{\epsilon}, \varphi v>\right|+\left|\int_{A} S_{\epsilon} \partial_{t} \varphi v d x d z\right| \leq c\left\|S_{\epsilon}\right\| \mathcal{V}\|v\|_{H^{1}(A)} .
$$

Therefore, $\partial_{t}\left(\varphi S_{\epsilon}\right)$ is bounded in $L^{2}\left(t_{0}, t_{1}, H^{-1}(A)\right)$ and, up to a subsequence, $\varphi S_{\epsilon}$ converges a.e. in ]$t_{0}, t_{1}[\times A$.

Since $\widetilde{\widetilde{Q}}$ is a countable reunion of sets of form $] t_{0}, t_{1}\left[\times A^{5}\right.$, by a diagonal extraction, it is possible to consider that $S_{\epsilon}$ converges a.e. to $S$ in $\widetilde{\widetilde{Q}}$. Then, since $\bar{\mu}=0$ outside $\widetilde{\widetilde{Q}}$, one is able to conclude that $\bar{\mu}\left(., S_{\epsilon}\right) S_{\epsilon}$ converges a.e. to $\bar{\mu}(., S) S$ and that $\chi=\bar{\mu}(., S) S$ (cf. J. L. Lions [23] Lemma 1.3 p.12). Then, the result of existence of a solution holds.

\footnotetext{
${ }^{5}$ Note that if $O \subset \mathbb{R}^{n}$ is open (for us, $O=\widetilde{\widetilde{Q}}, n=d+1$ ) and $O_{Q}=O \cap \mathbb{Q}^{n}$ then $O=\cup_{a \in O_{Q}} B\left(a, d\left(a, O^{c}\right)\right.$ ) where $O^{c}$ is the complementary on $O$. Indeed, if $b \in O$, there is $\gamma>0$ with $B(b, \gamma) \subset O$ and there is $a \in O_{Q}$ with $d(a, b)<\gamma / 2$. Then, $B(a, \gamma / 2) \subset B(b, \gamma) \subset O$ and $\gamma / 2 \leq d\left(b, O^{c}\right)$. Thus $b \in B\left(a, d\left(a, O^{c}\right)\right)$.
} 


\section{Numerical simulations}

The simulations have been obtained by the way of a classical control volume finite element method (CVFE) in order to conserve the mass of glass eel in the discretization. But, in our case the shape of the mesh changes over time in the $z$ direction since the position of the river surface is time-dependent. A first idea could be, as in the mathematical analysis, to work in a stationary domain by the introduction of a fictive complementary domain, but the size of the domain will increase the number of meshes. To take into account this vertical variation, the vector $\boldsymbol{V}$ is corrected by $\boldsymbol{V}=(a(t, x),-b(t, x, z)-\widetilde{v})$, where $\widetilde{v}$ is related to the deformation speed of the mesh according to the Arbitrary Lagrangian-Eulerian (ALE) method (cf. [15]). This strategy allows a total conservation of the glass eel mass; this was not achieved in the numerical models described in [28].

The following simulations have been obtained with real condition data observed during the fishing season November 1999 to March 2000.

A one-dimensional hydrodynamic module calculates the current speeds and the heights of water in the river. It is based on the Saint-Venant equation and uses concrete downstream and upstream flows measured by Ifremer during this period. The turbidity, the days, the nights and the different type of moons are also given. The horizontal domain is from the Adour mouth to after "Bec des Gaves", corresponding to $35 \mathrm{~km}$; the vertical part is in meters ${ }^{6}$. The results of the simulation are compared to the one obtained in the fishery-zones. They give a better behavior than the one of paper [28] and illustrate the improvements taken into account in the revision of the model.

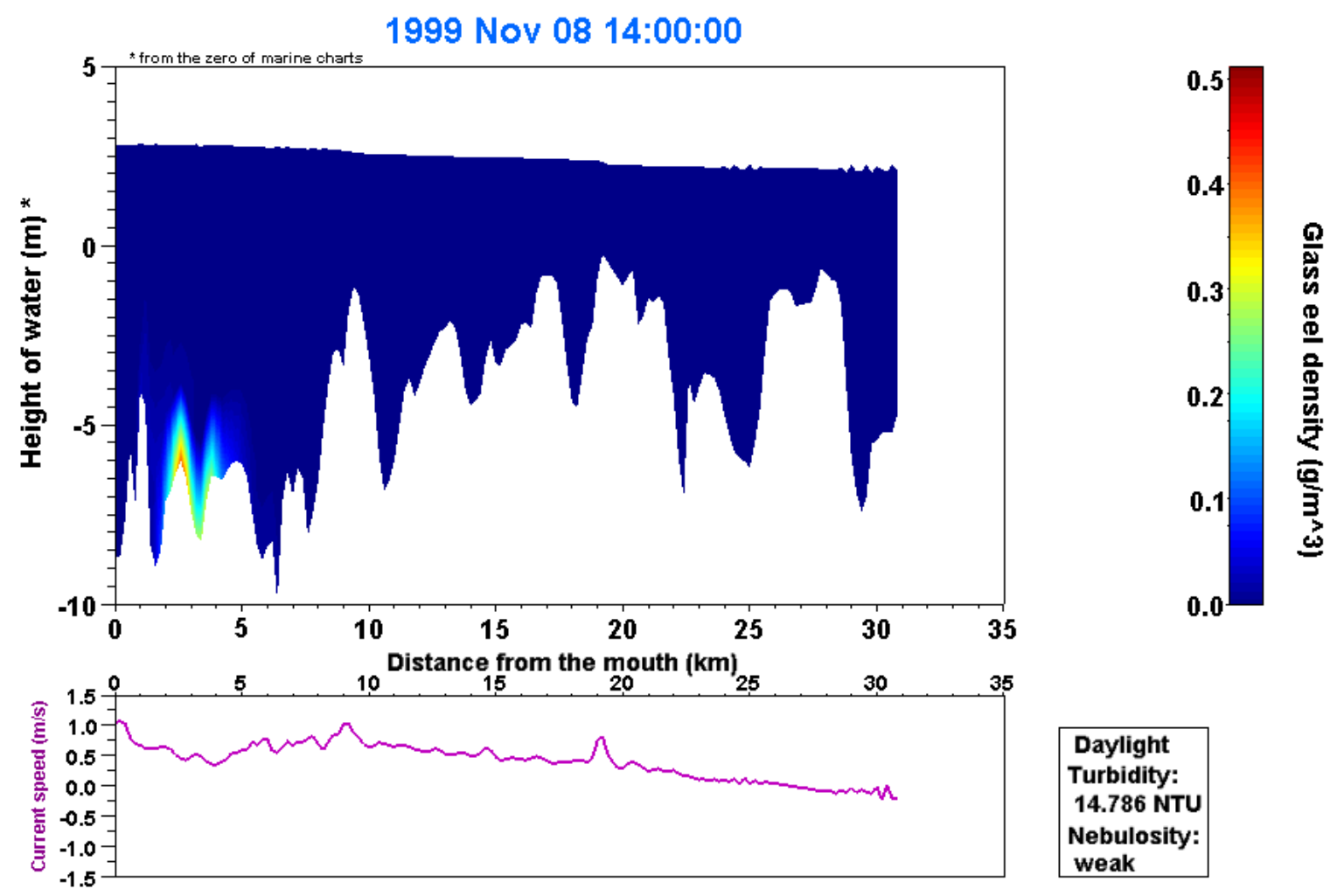

Figure 2. Daylight, $u>0$

\footnotetext{
${ }^{6}$ This scale difference explains the surprising variations of the river bottom.
} 


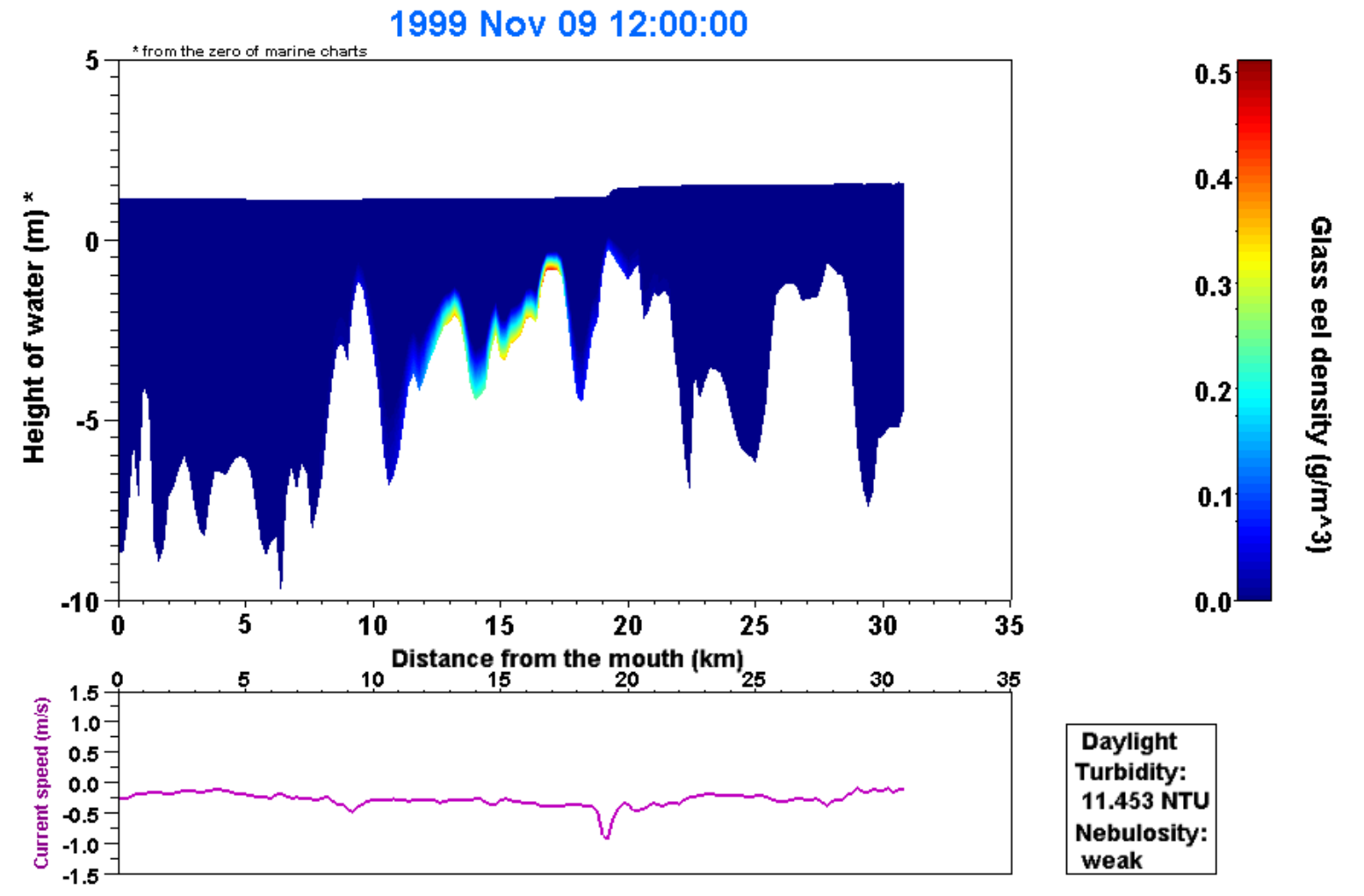

FiguRE 3. Daylight, $u<0$ 


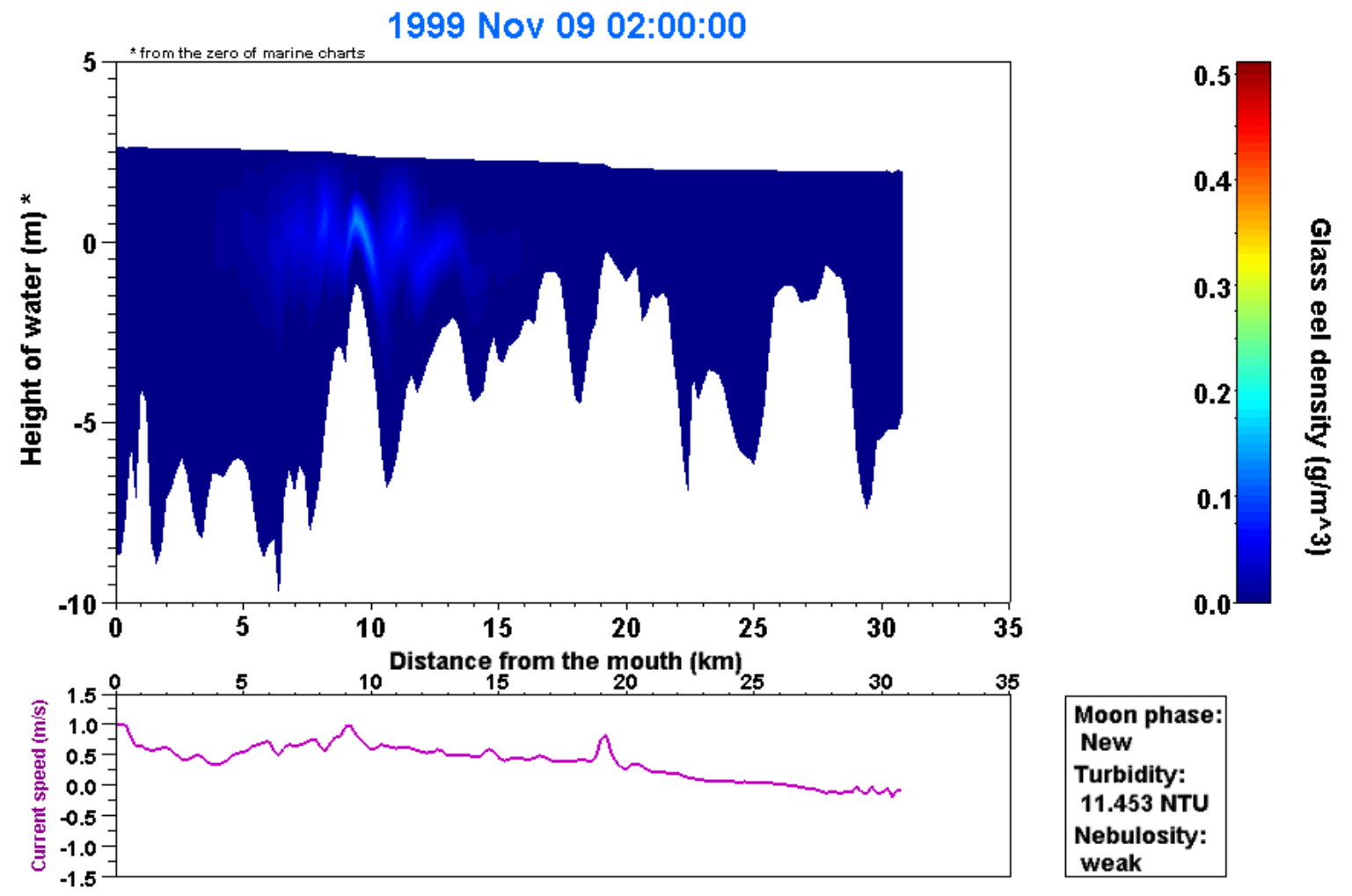

FiguRE 4. Night, $u>0$

In Figure 2, the current is favorable to the migration. A cloud of glass eels has just came into the domain by the boundary $\Gamma_{L}$. But it is 14 oÕclock, the light scares the elvers and they will remain at the bottom. It is the same, one day after in Figure 3. It is 12 oÕclock and the current is negative.

In Figure 4, the current is favorable to the migration and it is night (2 oÕclock). The glass eels come back up towards the surface and diffuse in the river. In Figure 5 , it is night ( 21 oÕclock) and the currents is negative. If $u<\hat{u}$, they remain at the bottom, else they can still diffuse horizontally while diving to the bottom.

Acknowledgements. This study was supported by the EU through the EELIAD project.

The authors would like to thank the referees whose many suggestions and remarks helped to improve the manuscript. 


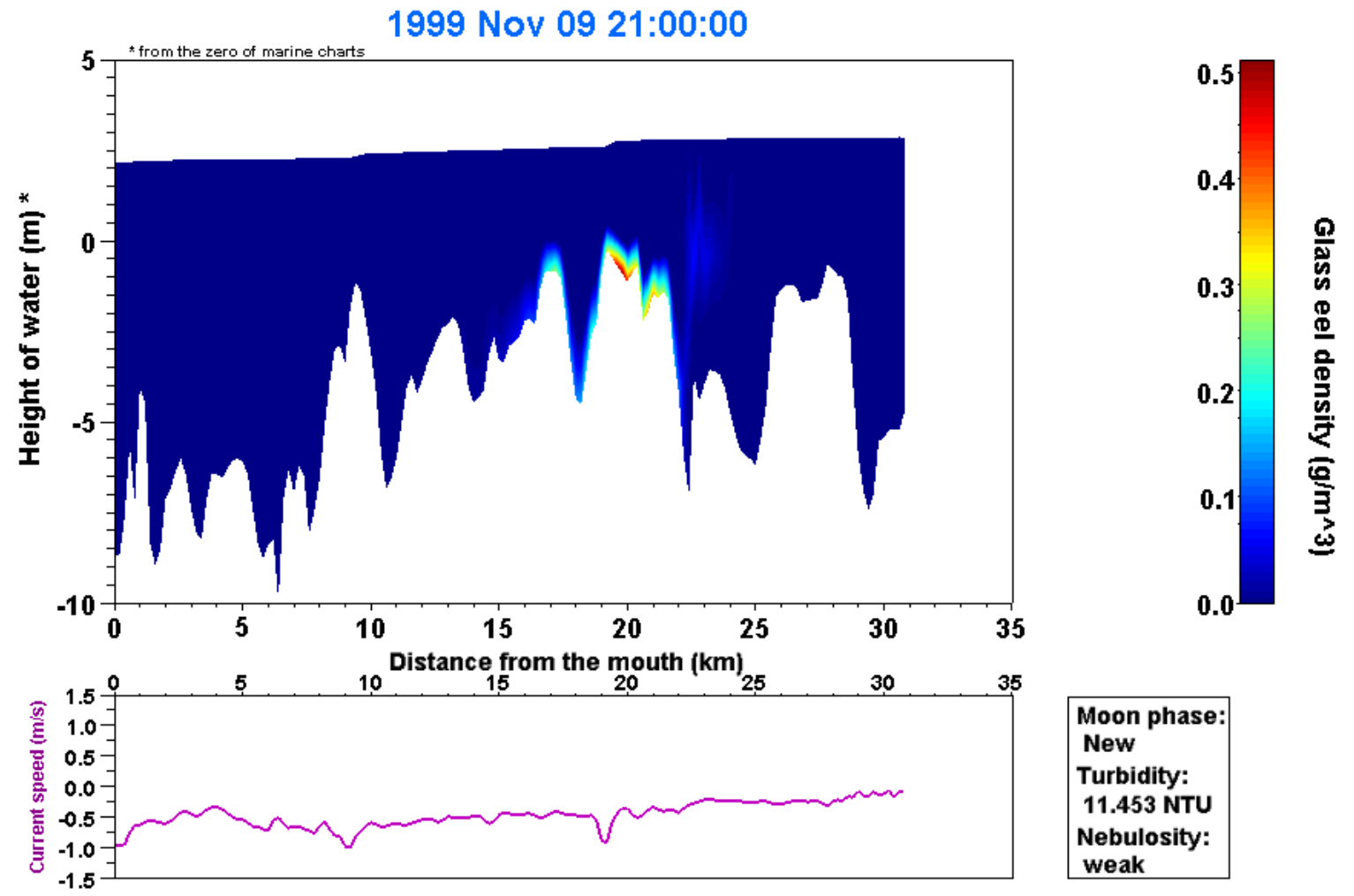

FiguRE 5. Night, $u<0$

\section{References}

[1] Adimurthi, S. Mishra, G.-D. Veerappa Gowda. Explicit Hopf-Lax type formulas for Hamilton-Jacobi equations and conservation laws with discontinuous coefficients. J. Differential Equations, 241 (2007), No. 1, 1-31.

[2] G. Aguilar, L. Lévi, M. Madaune-Tort. Nonlinear multidimensional parabolic-hyperbolic equations. Proceedings of the 2006 International Conference in honor of Jacqueline Fleckinger, Electron. J. Differ. Equ. Conf., 16 (2007), 15-28.

[3] A. Ambrosetti, A. Malchiodi. Nonlinear analysis and semilinear elliptic problems. Cambridge University Press, Cambridge, 2007.

[4] B. Andreianov, K. H. Karlsen, N. H. Risebro. A theory of $L^{1}$-dissipative solvers for scalar conservation laws with discontinuous flux. Arch. Ration. Mech. Anal., 201 (2011), No. 1, 27-86.

[5] A. Bardonnet, V. Bolliet, V. Belon. Recruitment abundance estimation: Role of glass eel (anguilla anguilla l.) response to light. Journal of Experimental Marine Biology and Ecology, 321 (2005), No. 2, 181-190.

[6] C. Bardos. Problèmes aux limites pour les équations aux dérivées partielles du premier ordre à coefficients réels; théorèmes d'approximation; application à l'équation de transport. Ann. Sci. École Norm. Sup., 3 (1970), $185-233$.

[7] M. Bendahmane, K. H. Karlsen. Anisotropic doubly nonlinear degenerate parabolic equations. Proceedings of ENUMATH 2005: Numerical mathematics and advanced applications, Springer, (2006), 381-386.

[8] S. Berres, R. Bürger, H. Frid. Neumann problems for quasi-linear parabolic systems modeling polydisperse suspensions. SIAM J. Math. Anal., 38 (2006), No. 2, 557-573.

[9] V. Bolliet, P. Lambert, J. Rives, A. Bardonnet. Rhythmic swimming activity in anguilla anguilla glass eels: Synchronisation to water current reversal under laboratory conditions. Journal of Experimental Marine Biology and Ecology, 344 (2007), No. 1, 54-66.

[10] J. Carrillo. Entropy solutions for nonlinear degenerate problems. Arch. Ration. Mech. Anal., 147 (1999), No. 4, $269-361$.

[11] G.-Q. Chen, K. H. Karlsen. $L^{1}$-framework for continuous dependence and error estimates for quasilinear anisotropic degenerate parabolic equations. Trans. Amer. Math. Soc., 358 (2006), No. 3, 937-963.

[12] G.-Q. Chen, B. Perthame. What is ... a kinetic solution for degenerate parabolic-hyperbolic equations ? Notices Amer. Math. Soc., 57 (2010), No. 6, 737-739.

[13] R. Dautray, J.-L. Lions. Analyse mathématique et calcul numérique pour les sciences et les techniques. Masson, 1988. 
[14] M. de Casamajor, P. Prouzet, P. Lazure. Identification des flux de civelles (anguilla anguilla) à partir des relations d'allométrie en fonction des conditions hydrodynamiques de l'estuaire de l'Adour. Aquatic Living Resources, 13 (2001), No. 6, 411-420.

[15] J. Donea, A. Huerta, J.-P. Ponthot, A. Rodríguez-Ferra. Arbitrary Lagrangian- Eulerian methods in Encyclopedia of computational mechanics, Vol. 1. John Wiley \& Sons Ltd., Chichester, 2004.

[16] J. Droniou. Non-coercive linear elliptic problems. Potential Anal., 17 (2002), No. 2, 181-203.

[17] G. Gagneux, M. Madaune-Tort. Analyse mathématique de modèles non linéaires de l'ingénierie pétrolière, SpringerVerlag, Berlin, 1996.

[18] F. Gastaldi, A. Quarteroni, G. Sacchi Landriani. Coupling of two-dimensional hyperbolic and elliptic equations. Comput. Methods Appl. Mech. Engrg., 80 (1990), No. 1-3, 347-354.

[19] F. Gastaldi, L. Gastaldi. On a domain decomposition for the transport equation: theory and finite element approximation. IMA J. Numer. Anal., 14 (1994), No. 1, 111-135.

[20] D. Gilbarg, N.-S. Trudinger. Elliptic partial differential equations of second order. Springer-Verlag, Berlin, 2001.

[21] J. Jimenez, L. Lévi. A mathematical analysis for some class of hyperbolic-parabolic problems. Adv. Math. Sci. Appl., 20 (2010), No. 1, 51-75.

[22] S.-N. Kružkov. First order quasilinear equations with several independent variables. Mat. Sb. (N.S.), 81 (1970), No. 123, $228-255$.

[23] J.-L. Lions. Quelques méthodes de résolution des problèmes aux limites non linéaires. Dunod, Paris, 1969.

[24] J.-L. Lions, E. Magenes. Problèmes aux limites non homogènes et applications I. Dunod, Paris, 1968.

[25] M. Odunlami. "GlassEel2D". A software to simulate glass eel behavior in estuaries, University of Pau, https://redmine.univ-pau.fr/projects/glasseel2D, 2011.

[26] O. Pardo. Contribution à l'étude et à la modélisation d'un modèle de convection-diffusion dégénéré : application à l'étude du comportement migratoire des civelles dans l'estuaire de l'Adour. PhD thesis, Université de Pau, 2002.

[27] P. Prouzet and EELIAD partners. Personal communications. IFREMER and http://www.eeliad.com/, $2009-2012$.

[28] P. Prouzet, M. Odunlami, E. Duquesne, A. Boussouar. Analysis and visualization of the glass eel behavior ( anguilla anguilla) in the adour estuary and estimate of its upstream migration speed. Aquatic Living Resources, 22 (2009), $525-534$.

[29] J.-E. Roberts, J.-M. Thomas. Mixed and hybrid methods. In Handbook of numerical analysis II. North-Holland, Amsterdam, 1991.

[30] G. Vallet, P. Wittbold. On a stochastic first-order hyperbolic equation in a bounded domain. Infinite Dimensional Analysis, QuantumProbability, 12 (2009), No. 4, 1-39. 